\title{
Pathogenesis, Diagnosis and Treatment of Vaginitis and Cervicitis in Clinical Practice
}

\author{
${ }^{1,}$ MurtazaMustafa, ${ }^{2,}$ Bendaman B Yanggau, ${ }^{3}$ HelenLasimbang \\ ${ }^{1,2,3,}$ Faculty of Medicine and Health Sciences, University Malaysia Sabah, KotaKinabalu, \\ Sabah,Malaysia.
}

\begin{abstract}
Vulvovaginitis results with the loss of Lactobacillus-dominated normal vaginal flora,with the sexual activity pathogenic organisms are introduced into the vagina. Lactobacillus maintain normal vaginal $\mathrm{pH}$ of 3.5, and production of hydrogen peroxide which is bactericidal. Primarily vaginitis include three infections, trichomonaiasis,vulvovaginalcandidiasis, and bacterial vaginosis $(B V)$,desquamative inflammatory vaginitis(DIV), associated with estrogen deficiency. Cervicitis primarilyendocervicitis caused by sexually transmittedpathogens.Vaginal pathogens include: Candidaalbicans, Trichomonasvaginalis,Neisseriagonorrhoeae,C.trichomatis,Gardnerrelavaginalis.Drugs of choice for candidiasis, Fluconazole 150mg orally in a single dose with vaginal preparations, and metronidazole for trichomoniasis.Patienthistory, complete examination, and laboratory tests are essential for diagnosis of vaginitis or cervicitis.
\end{abstract}

KEYWORDS:Vaginitis,Trichomoniasis,Cervicitis,Treatment.

\section{INTRODUCTION}

Vulvovaginal symptoms are common and frequently result in encounter of patients with healthcaresystem,including use of folk remedies, purchase of over-the counter (OTC) pharmaceuticals, and presentations to healthcare providers [1].Cervicitis may be infectious or noninfectious is primarily an endocervicitis caused by Neisseria gonorrhoeae,C.trachomatis or both of these sexually transmitted pathogens [2].The normal vaginal secretions are a physiologically important biomass.Vaginal cells contain glycogen and are continually shed into the lumen of vagina.As the cells autolyze,glycogen deploymerizes to glucosewhich serves as energy source for bacteria known as lactobacilli.Lactobacillus crispatusand lactobacilli jenseniiare the predominant species [3].Lactobacilli metabolize glucose to lactic acid, which results in a normal vaginal $\mathrm{pH}$ of 3.5 to 4.6.Lactobacilli also produce hydrogen peroxide, which is bactericidal alone and highly bactericidal in combination with physiologic amounts of myeloperoxidase and chloride [4].Loss of the normal Lactobacillusdominated vaginal flora increases the likelihood of exogenous infection after exposure to sexually transmitted pathogens, as well as the risk of endogenous infection in association with pregnancy and gynecologic surgery[5,6].In addition to normal secretions, the differential diagnosis of vaginal discharge primarily includes three infections,e.g.trichomoniasis, vulvovaginal candidiasis and bacterial vaginosis (BV);an idiopathic condition known as desquamative inflammatory vaginitis (DIV);cervicitis, both infectious and noninfectious, andvulvovaginitis associated with estrogen deficiency [7].Patient medical history should include all of the usual gynecologic parameters, including menstrual history, pregnancies,contraception,sexual preference(sexual orientation),past and present sexual relationships, and prior genitourinary infections, underlyingmedical conditionssuch as allergies, diabetes, malignancies, andimmunodeficiency syndrome (primarily human immunodeficiency virus (HIV) disease) that might be associated with vulvovaginal disease [7].Common pathogens

albicans,Trichomonasvaginalis,Neisseriagonorrhoeae,C.trichomatis,Gardnerellavaginalis and others. Treatment of candidiasis, uncomplicated or complicated is different. Effective drugs for candidiasis include Fluconazole $150 \mathrm{mg}$ orally in a single dose with vaginal preparationsand metronidazole for trichomoniasis. The paper reviews pathogenesis, diagnosis and therapy of vaginitis and cervicitis.

\section{CANDIDA VULVOVAGINITIS}

Etiologic agent and pathogenesis

Candida albicans can be isolated from about $80 \%$ to $90 \%$ of patients with vulvovaginal candidiasis,and the other yeasts account for up to $20 \%$ of cases [8].Candida tropicalis is isolated from about $1 \%$ to $5 \%$ and may be associated with a higher rate of recurrence after standard treatment [9].Candida (formerly Torulopsis) glabrata accounts for about $10 \%$ of vaginal yeast isolates [8].Symptomatic vaginitis caused by this organism is associated with less intense itching and dyspareunia than caused by other Candidaspecies, but organism may be 
harder to eradicate with standard therapies[10].the relative incidence of vaginitis caused by fungi other than C.albicans appears to be increasing. Non-albicans infections are associated with recurrent disease (accounting $21 \%$ of recurrent vs $12 \%$ of initial infections) and with HIV infection (22\% of infections in HIV-positive women vs $12 \%$ in HIV-negative women), especially in HIV-infected women who receive prophylaxis with imidazoles[9].It is thought that the widespread use of topical antifungal agents, especially short courses ,may contribute to selection for non-albicans yeasts, which are less susceptible to these agents than is C.albicans.Cases of vaginitis caused by Saccharomyces cerevisiae have been reported and may be associated with baking[11].Some workers have estimated that $75 \%$ ofadult women will suffer at least one episode of vulvovaginal candidiasis during their life time[12].Inhibition of normal bacterial flora by antibiotics favors the growth of yeasts, althoughsymptomatic cases are seen after the use of antimicrobials that do not suppress lactobacilli.Vulvovaginal candidiasis sometimes occurs after antimicrobial treatment of trichomonas or BV [12].Growth of yeasts is apparently favored by high estrogen levels, although such levels also promote the growth of lactobacilli[13].The prevalence of carriage of Candida is higher among users of oral contraceptives than woman using other methods of birth control. The mechanism of this estrogenic predisposition is unclear [14].Vulvovaginal candidiasis is associated with poorly controlled diabetes mellitus, and tight glycemic control decreases the frequency symptomatic infection [15].However, testing for diabetes in women with recurrent vulvovaginal candidiasis is not cost effective [14].It has been suggested that tight insulating clothing predisposes to vulvovaginal candidiasis by increasing vulval warmth and moisture. In prospective studies, a higher prevalence of candida carriage and higher concentrations of organisms were found in women who wore tight rather loose clothing [15].Impairment of phagocytic or of cell-mediated immunity (e.g.transplantation,chemotherapy)alsopredisposes to vulvovaginalcandidiasis.Some authoritiesbelieve that women with HIV infection develop vulvovaginal candidiasis more often than HIV-negative women do, especiallyif they have low CD4 T-cell count[16]

The contribution of sexual transmission is poorly defined.Vovovaginal candidiasis increases in incidence with onset of sexual activity, but incidence is also increased by the use of oral contraceptives, the contraceptive sponge, or the intrauterine device, any of which might coincide with sexual activity[17,14,18]Having multiple sexual partners is not associated with higher incidence of Candida infection.Most women who present with vulvovaginal candidiasis have no predisposing illness or medications [7] The mechanism by which Candidaproduces disease is not well defined. Although it is postulated that differences in virulence must exist, strains isolated from symptomatic women are not demonstrably different from isolates from asymptomatic carriers [14,19] Filamentous forms(hyphae and pseudohyphae) are associated with active disease.Pseudohyphae have been observed to penetrate vaginal epithelial cells, and they are more adherent to cells than are budding yeasts(blast pores)[20-22]. Adherence appears to be an important pathogenic feature of Candida spp,and sublethal concentrations ofantifungal agents may ameliorate disease by reducing adherence [23,24].The severity of symptoms in vulvovaginal candidiasis is not directly related to the number of yeast cells present. Indeed very small numbers of yeasts may be present in vaginal material recovered from highly symptomatic women [14].An immunologic reaction has been suggested as the mechanism for symptomatic disease in such women, and one small series suggested that desensitization may decrease the frequency of symptomatic episodes[25,26].

\section{CLINICAL PRESENTATION}

Patients with candida vulvovagnitis generally complain of perivaginalperuritis,often with little or no discharge. Dysuria is occasionally noted and is likely to be perceivedas vulvar rather than uretheral.The labia may be pale or erytheomatous. Shallow,radial,linear ulceration, especially in the posterior portion of the introitus,,are common.Excoriations caused by scratching are often present, just beyond the main area helped diagnostically. The vaginal walls may erythematous.Candidal discharge is classically thick and adherent.However, it may be thin and loose, resembling the discharge of other vaginitides[7].

Diagnosis of Candida vaginitis : Vaginal $\mathrm{pH}$ is usually normal. There is no odor when the vaginal secretion are mixed with $10 \% \mathrm{KOH}$ (whiff test).Microscopic examination of vaginal material in saline or in $10 \% \mathrm{KOH}$ may disclose budding yeasts or mycelia. In the symptomatic patient with a diagnostic microscopic examination, fungal cultures are not needed. Microscopic examination of vaginal secretion is incompletely sensitive, however.Accordingly cultures may be helpful to secure the diagnosis in a patient who has a compatible clinical presentation and a negative microscopic examination. It is usually expedient to treat such a patient with antifungal agents while awaiting culture results. Cultures are useful if empirical treatment produces no response [7]. 
Treatment of Candida vaginitis:Therapy of vulvovaginal candidiasis is based on clinical diagnosis e.g.uncomplicated or complicated infection. Uncomplicatedvulvovaginal candidasis is a sporadic infection with C.albicans, of mild to moderate severity, in womenwithout a history of recent candidacies and without underlying illness such as HIV disease or diabetes mellitus. Most patients have uncomplicated disease, and most cases respond to treatment with short courses of vaginal preparations containing nystatin ,miconazole, clotrimazole, butconazole ,terconazole , and tioconazole. All except nystatin and terconazole containing products are available without prescription[27].In uncomplicated disease, there does not appear to be any difference in efficiency related to dosage from vaginal tablets, suppositories, ointment, creams or length of treatment $(1,3,7$ or 14 days). Oral fluconazole in a single $150 \mathrm{mg}$ dose is as effective as aforementioned vaginal medications [28].

\section{Etiologic agent and pathogenesis}

\section{TRICHOMONIASIS}

Trichomoniasis is caused by the protozoan Trichomonasvaginalis.It is classic exogenous sexually transmitted infection, like gonorrhea and chlamydial infection. The organism is not normally present in the vagina.Transmission almost always occurs through sexual contact. After an incubation period of few days, patients develop a purulent discharge associated with varying degrees ofvulvalirritation,dysuria,anddysparunia.An abnormal odor is often present usually signifying concomitant BV [7].

Diagnostic tests:Most patients present with vestibular and vaginal erythema and a purulent vaginal discharge. A minority of patients' manifest characteristic mucosal capillary dilation, which gives the mucosa a strawberry appearance [7] .Vaginal $\mathrm{pH}$, is always greater than 4.5.A positive whiff test is not unusual. The vaginal wet preparation contains abundance of leukocytes and motile flagellated. The wet preparation have sensitivity of 60\% to70\% in symptomatic patients [29].Non-culture tests includeOSOM Trichomonas Rapid Test (Genzyme),Affirm VOIII(Becton Dikenson),and XenostripTv(Xenotype Diagnostic)These tests have sensitivity that approaches that of culture[30].Sensitive and specific nucleic acid amplification tests have also been developed [31].Diagnostic tests in which organisms are no longer motile may lack specificity. A common clinical problem is the women with no epidemiologic evidencefor a sexually transmitted condition who has trichomonads visualized on a cervical cytological examination. In some such cases, the cytologist may misread the smear [32].

Treatment of Trichomoniasis : Metronidazole and tinidazole are the only effective agents that are approved by the FDA for the treatment of tricomoniasis.A single2-g oral dose of metronidazole or tinidazole can be prescribed.Alternatively,500 mg oral metronidazole be given twice daily for 7 days. Controlled studies have failed to show any important advantage of the 7-day regimen. A single- dose regimen if administered under direct observation in the office or in the clinic has the obvious advantage of 100\% compliance. Because the trichomoniasis is almost always sexually transmitted, treatment with metronidazole of recent sexual partners. Regardless of their symptoms, is an integral part of management [33].For patients who cannot tolerate metronidazole at this dosage level and for those who do not respond to it,tinidazole has better in-vitro efficacy, is better tolerated than metronidazole, and has cured most patients with metronidazole resistant trichomoniasis[34].Trichomonasvaginalis organisms have been shown to be estrogen dependent in vitro and in vivo[35,36].In one report, discontinuation of estrogen replacement treatment in a postmenopausal woman was associated with resolution of vaginal trichomaniasis [37].Human immunodeficiency virus(HIV) infections has no effect on the incidence or prevalence of trichomoniasis or on persistence or recurrence [38].Metronidazole has traditionally been avoided during, pregnancy because of largely theoretical concerns about mutagenicityandoncogeneticity.However,studiesand meta analyses have not demonstrated a consistent association between metronidazole use during pregnancy and teratogenic or mutagenic effects in infants[39].

Etiologic agent and pathogenesis

\section{BACTERIAL VAGINOSIS}

Bacterial vaginosis (BV) was initially described in sexually active women, and it is common in populations with high prevalence of sexually transmitted diseases [40].BV was first described by Gardner et al. for the women who present to their physician with vaginalsymptoms have a specific condition[41].Discharge is often present at the introitus and visible on the labiaminora .The labia and vulva are not erythromatous or edematous. On speculum examination, the vaginal walls appear uninflamed. The vagina usually contains a grayish, thin homogenous discharge. A pungent odor may be noted by the examiner. The endocervix is unaffected by the process, and any cervical discharge should be physiological. The presence of a purulent cervical discharge may result from coincident gonococcal or chlamydial infection [42].Abnormalities on 
bimanual examination is unusual in uncomplicated $\mathrm{BV}$ and should prompt a search for other pathologic process. That is an increased risk of endomerititis and salpingitis among women with BV [43].Microscpic examination of vaginal discharge in BV characteristically reveals flora of coccobacilli, Gardnerellavaginalis (formerly Haemophilusvaginalis). Although Gardner regularly produced BV by inoculating fresh vaginal discharge from patients with BV into vagina of healthy volunteers, inoculation of pure culture of G.vaginalis was far less likely to produce disease [43].The explanation of all these observations is that G.vaginalis is not the single cause of BV.BV is usually a synergistic infection involving not only G.vaginalis but also other microorganisms. The total number of organisms dramatically increased in the vaginas of women with BV[44].Hydrogen peroxideproducing lactobacilli dominate the normal vaginal flora, and appear to protect against exogenous infection. Some workers believe that an undefined change in the vaginal milieu permits the replacement of protective H20-producing lactobacilli with G.vaginalisand other microorganisms [3].Some investigators have linked douching to BV [45]. Women who have BV are at increased risk for development of infection with herpes simplex virus type2,N.gonorrhoae and C.trachomatis [46,5].

Diagnosis of BV:The patient is most likely to complain of odor and of discharge which tends to be gray and homogenous. Theodor is best described a "fishy" is caused by amines such asmethylamine.These amines volatize at increased $\mathrm{pH}$, which explains the propensity of the patient to notice the odor when the selections are more(e.g.during menses, after intercourse .Vulvovaginal irritation is not usually prominent symptom hence the use of term vaginosis rather vaginitis[7].Microscopic examination of vaginal secretions suspended in $0.9 \%$ NCL reveals few leukocytes and many small bacilli. These bacilli tend to coat vaginal epithelial-so called "clue cells", name by Herman Gardner because they provided a clue to the diagnosis of this condition [7]. Symptoms of BV include: (a) homogenous vaginal discharge,(b)fishy vaginal odor,(c)during menstruation \& after intercourse,(d)minimal itching or irritation[7].Criteria for the diagnosis of BV include (i)homogenous vaginal discharge, (ii)vaginal $\mathrm{pH}$ greater than 4.6,(iii)positive whiff test with $10 \% \mathrm{KOH}$,(iv)clue cells.Asmel and colleagues [47],suggested at least three of four listed criteria be present for the diagnosis of BV to be made. Criteria developed by Nugent and colleagues for the diagnosis of BV with use of the Gram stain [48].Highly sensitive tests such as polymerase chain reaction (PCR) directed at G.vaginalis are not useful due to high prevalence of G.vaginalis among health individuals [49].

Treatment of BV :The primary regimen for the treatment of BV is oral metronidazole,500 $\mathrm{mg}$ twice a day for 7 days.A single 2.0-g dose of metronidazole such as used to treat trichomoniasis is less effective and is not recommended[50].Vaginal preparations containing $0.75 \%$ metronidazole gel or $2 \%$ clindamycin cream or ovules containing $100 \mathrm{mg}$ od clindamycin are effective and have few systemic effects [51-53].Treatment failure occur fairly commonly, presumable because normal Lactobacillus-dominated flora fails to become established after anaerobes and other components of BV flora have been reduced in number with use of antimicrobial agents[54].Recent studies has shown that persistence of G.vaginalis in biofilims on the vaginal wall may associated with treatment failure [55].

\section{Etiologic agent and Pathogenesis}

\section{CERVICITIS}

Infectious cervicitis is primarily an endocervicitis caused by N.gonorrhoeae, C.trichomatis or both of these sexually transmitted pathogens[2].Primary herpes simplex virus infection can cause endocervicitis,but seldom occurs without vulvar lesions that make the diagnosis obvious.Mycoplasmagenitalium has recently been associated with cervicitis[2]. Noninfectious cervicitis is usually is ectocervicitis in which there is inflammation in an ectropion[7].

Diagnosis and treatment : The patient usually complains of a purulent vaginal discharge.Themucopurulent secretions are not irritating, so there is no vulvar discomfort or introitaldyspareunia.Becusegonococcal or chalamydial infection can involve the urethera,endometrium,oradnexa,there may be dysuria,abnormal uterine bleeding, lower abdominal pain, or pelvic dyspareunia[7].Findings on examination of vulva and the vaginal mucosa are usually normal. In infectious endocervicitis, the purulent secretions can be seen to flow from the endocervicalcanal;in noninfectious cervicitis, the purulent secretions can be seen to emanate from the ectropion[7].Vaginal $\mathrm{pH}$ may be elevated. There is no odor when secretions are mixed with $10 \% \mathrm{KOH}$.Wet preparations of vaginal secretions contain many leukocytes.Vaginal cells are mature.Gram- stained smears of cervical secretions confirm the presence of many leukocytes,and in gonococcalinfection,may contain intracellular cocci.Culture or non-culture for N.gonorrhoeae and C.trachomatis should be performed [7].Patients who have positive tests for sexually transmitted bacteria should be treated with regimens recommended for the etiologicagent.[33]If tests for gonococcal and chlamydia are negative antimicrobial treatment will not likely to be of benefit.If the volume of secretions from an ectropion is bothersome, destruction of the endocervical 
mucosa of the ectropion with cryotherapy may allow the endocervix to become reepithelialized with squamous epithelium, with a resultant diminution of the volume of the ectrocervical secretions [7].

\section{DESEQUAMATIVE INFLAMMATORY VAGINITIS}

\section{Etiologic agent and pathogenesis}

DIV is an unusual condition of unknown cause.It mimics estrogen deficiency vaginitis and trichomoniasis but usually occurs in women of reproductive age who have normal hormonal function and no evidence of any sexually transmitted conditions [7].This disorder sometimes occurs in premenopausal women or after pregnancy suggesting a role for changes in the level of estrogen in its etiology [56].

Diagnosis and treatment:The main characteristics of DIV include: (a) purulent discharge (b) vulvar discomfort (c) dyspareunia,and findings are mucosal erythema, purulent secretions, and parabasalcells. The vaginal $\mathrm{pH}$ often elevated to greater than 4.6.There is no odor when the vaginal secretions are mixed with $10 \% \mathrm{KOH}$.The saline preparation contains many leukocytes. Most of the vaginal epithelial cells are immature parabasalcells [56].The primary regimen for the treatment of DIV is topical corticosteroids and topical boric acid provide systematic relief. Relapse is predictable after these agents are discontinued .By far the most effective treatment for this condition is $2 \%$ clindamycin vaginal cream is inserted into the vagina at bedtime for 14 days [56].

\section{Etiologic agent and pathogenesis}

\section{ESTROGEN DEFICIENCY VAGINITIS}

Estrogen deficiency vaginitis is seen in postmenopausal women and in younger women who have become estrogen deficient because of disease or because of treatment with pharmaceuticals that interfere with the production of estrogen [57].This condition can also be seen during breastfeeding because of an effect of prolactin on estrogen production. [58]. Without estrogen vaginal mucosa thins. Glycogen is decreased and as a result lactobacilli no longer dominate the vaginal flora. Thinning of the mucosa may result in vulvar discomfort and introital dyspareunia. The thin vaginal mucosa may become infected with enteric organisms and others that are able to colonize the vagina in the absence of lactobacilli[7].

Diagnosis and treatment : Vaginal secretions, if present may be purulent.Vaginal $\mathrm{pH}$ is elevated. There is no odor when the secretions are mixed with 10\% KOH.Microscopic examination of the secretions discloses immature (parabasal)vaginal cells with or without leukocytes. Vaginal cultures contain a variety of enteric and other bacteria [7].The treatment involves estrogen replacement or cessation of antiestrogenic drugs or breastfeeding.Topical antibacterial agents containing sulfonamides or clindamycin may improve symptomatic vaginitis and lubrication agents may relieve vaginal dryness and dyspareunia[7].

\section{CONCLUSION}

A complete history, "whifftest",pHmeasurement of vaginal secretions, and carefulevaluation will help the diagnosis and treatment of vaginitis and cervicitis.

\section{REFERENCES}

[1] McCormak WM jr,Zinner SH, MacCormakWM.The incidence of genitourinary infections in a cohort of healthy women.SexTransm Dis.1994;21:63-64.

[2] MarrazoJM,MartinDH.Management of women with cervicitis.Clin InfectDis.2007;44(Supp3):5102-5110.

[3] VallorAC,AntoninMA,HawesSE,etal.Factors -associated with acquisition of or persistent colonization by vaginal lactobacilli.Role of hydrogen peroxide production.J Infect Dis.2001;184:1431-36.

[4] KlebanofSI,HillierSi,EschenbachDA, etal.Control of the normal flora of the vagina H0-generating lactobacilli.J Infect Dis.1991;164:94-100.

[5] WiesenfieldHc,HillierSI,KrohnMA,et al. Bacterial vaginosis is a strong predictor of Neisseria gonorrhoeae and Chlamydia trachomatis infection .Clin InfectDis.2003;36:663-68.

[6] SoperDE,BumpRC,HurtWC.Bacterialvaginosis and trichomoniasis are risk factors for calf cellulitis after abdominal hysterectomy. Am J Obstet Gynecol.1990;63:1016-23.

[7] McCormakWM.Vulvovaginitis and cervicitis.In:MandelGL,BennetJE,DolinR.edsPrinciples and practice on infectious diseases $7^{\text {th }}$ ed .NewYork:Churchil Livingstone,2010:1495-1509.

[8] VerminskyJP,SelfMJ,ChadvickSG,etal.Survey of vaginal flora Candida species isolates from women of different age groups by use of species -specific PCR detection.JClin Microbiol.2008;46:1501-1503.

[9] SpinilloA,CapuzzoE,GulminetiR,etal.Prevalence and risk factors for fungal vaginitis caused by non-albicansspecies.AmJ Obstet Gynecol.1997;176:138-141.

[10] Geiger Aw,FoxmanB,SobelJD.Chronicvulvovaginal candidiasis: Characteristics of women with Candida albicans,Cglabrata and no Candida genitourin.Med.1995;75:304-307.

[11] SobelJD,VazquezJ.LynchM,etal.Vaginitis due to Saccheromycescerevisiae:Epidemiology; chemical aspects; andtherapy.Clin Infect Dis.1995;16:95-99. 
[12] Agnew KJ,HillierSI.The effect of treatment regimens for vaginitis and cervicitis on vaginal colonization with lactobacilli.SexTransm Dis.1995;22:269-73.

[13] Larsen B.Vaginal flora in health and disease.ClinObstet Gynecol.1993;36:107-121.

[14] SobelJD.Pathogenesis and treatment of recurrent vulvovaginalcandidiasis.Clin Infect Dis.1992;14 (Suppl1):SI48-S153.

[15] No authors listed.Adverse events and their association with treatment regimens in the diabetes control and complications trial.Diabetes Care.1995;18:1415-27.

[16] SobelJD.Vulvovaginalcandidiasis:A comparison of HIV positive and negative womens.Int J STD AIDS.2002;13:358-62.

[17] Sobel JD. Vaginitis.NEngl J Med.1997;337:1896-1903

[18] GiegerAM,FoxmanB,Gillespie.The epidemiology of Vulvovaginalcandidiasis among university students.Am $J \quad P u b l i c$ Health.1995;85:1146-48.

[19] Odds FC.Genitalcandidiasis.ClinExp Dermato.1982;345:-54

[20] Odds FC.Candida and Candidiasis.Baltimore University Park:1979;4.

[21] Garcia Tamayo J,CastllaG,MartinezAI.Humangentitalcandidiasis:Histochemistry scanning and transmission electron microscopy.Acta Cytol.1982;26:7-14.

[22] Kimura IH,PearsallNN.Relationship between germination of Candida albicans and increased adherence in human buccal epithelial cells. Infect Immun.1980;28:164-68.

[23] King RD,LeeJC,MorrisAI.Adherence of Candida albicans and other candida species in mucosal epithelial cells.Int Immun.1980;27:667-74.

[24] SobelJD,MullerG.Ketaconazole in the prevention of experimental candida vaginitis.Antimicrob Agents Chemother.1984;25:28182 .

[25] WitkinSS,JeremiasJ,Ledger WJ.A localized vaginal allergic response in women with recurrent vaginitis.JAllergClin Immunol.1988;81:412-16.

[26] RiggD,MillerMM,MetzgerWJ.Recurrent allergic vulvovignitis: Treatment Candida albicans allergen immunotherapy.Am $J$ Obstet Gynecol.1990;162:332-36.

[27] Ferris DG,NyirjesyR,SobelJD,et al.Over-the-counter antifungal drug misuse associated with patient-diagnosed vulvovaginalcandidiasis. Obstet Gynecol. 2002;99:419-25.

[28] SobelJD,BrookerD,SteinGE,etal.Single oral dose fluconazole compared with conventional clotrimazole typical therapy of Candida albicans.Ann J ObstetGynecol.1995;72:1263-68.

[29] DemeoLR,DraperDI,MacGregorJA,etal.Evolution of a deoxyribonucleic acid probe for the detection of Trichomonasvaginalis in vaginal secretions.Am J Obstet Gynecol.1996;174:1339-42.

[30] HupportJS,MortensenJE,ReedJ,etal.sRapid antigen testing compares favorably with transcription-mediated amplification assay for the detection of Trichomonasvaginalis in young women.Clin Infect Dis.2007;45:194-98.

[31] Munson E,NapieralaM,OlsonR,etal.Impact of Trichomonasvaginalis transcription-mediated amplification-based analyte specificreagent testing in a metropolitan setting of high sexually transmitted diseases prevalence.JClinMirobiol. 2008; 46:3368-74.

[32] Lobo TT,Fejo G, CarvalhoSE, etal.A comparative evaluation of the Papanicolaou test for the diagnosis of trichomoniasis.SexTransm Dis.2003;30:694-99.

[33] Centers for Disease Control and Prevention. WorkowskiKA,BermanSM.Sexually transmitted diseases treatment guideline.2006.MMWR Recomm Rep.2006;55:1-94.

[34] Crowell AI,Sanders Lewis KA,SecorWE.In vitro metronidazole and tinidazole activities against metronidazole resistant strains of Trichomonasvaginalis.AntimicrobAgents Chemother.2003;47:1407-09.

[35] MartinottiMG,SaviaD,Effect of some steroid hormones on the growth of Trichomonasvaginalis.GBacteriolVirol Immunol.1985;78:52-59.

[36] Azuma T.A studyof the parasitizing condition of Trichomonasvaginalis with special reference to the relationship between estrogen and the growth of Trichomonasvaginalis.JJapnObstetGynec Soc.1968;15:168-72.

[37] Sharma R, PicheringJ,McCormakWM.Trichomoniasis in a postmanupausal women after discontinuation of estrogen replacement therapy.Sex Transm Dis.1997;24:543-45.

[38] Ca-UvinS,KoH,JameisonDJ,et al.Prevalence incidence and persistence or recurrence of traichomoniasis among human immunodeficiency virus (HIV)-positive woman and among HIV-negative women at high risk for HIV infection.Clin InfectDis.2007;34: 1406-1411.

[39] Caro-Paton T,CarvajalA,Martin de Diego J,et al. Is metronidazole teratogenic?.A meta-analysis.Br $J$ Clin Pharmacol.1997;44:179-82.

[40] FethersKA,FairleyCK,HockingJS,et al.Sexual risk factors and bacterial vaginosis:A systematic review and meta-analysis.Clin Infect Dis.2008;47:1426-35.

[41] Gardner HI,DukesCD.Haemophilusvaginalis vaginitis: A newly defined specific infection previously classified nonspecific"vaginitis.Am J Obstet Gynecol.1955; 69: 962-76.

[42] BrunhamRC,PaavonenJ,StevensCE, etal.Mucopurulentcervicitis: The ignored counterpart in woman of urethritis in men. $N$ Engl $J$ Med.1984;311:1-6.

[43] EschenbachDA.Bacterial vaginitis and anaerobes in obstetric gynaecologyic infection. Clin Infect Dis.1993;16(Suppl 4)5282-87.

[44] Spear GT,SikaroodiM,ZariffardMR, et al. Comparison of the diversity of the vaginal microbiota in HIV infected and HIV noninfected woman with or without bacterial vaginosis.J Infect Dis.2008;198:1131-40.

[45] BrotmanRM,GhanemNG,KlebanoffMA,etal.The effect of vaginal douching cessation on bacterial vaginosis:A pilot study.AmJ Obstet Gynecol.2008;198:628.e7

[46] NagotN,OuredraogoA,DeferMC,etal.Association between bacterial vaginosis and herpes simplex virus type-2 infection Implications for HIV acquisition studies. Transm Infect.2007;83:365-68.

[47] AmselR,Totten PA, Spiegel CA,etal.Nonpecific vaginitis Diagnostic criteria and microbial and epidemiologic association. Am J Med.1983;74:14-22.

[48] NugentRP,KrohnMA,HillierSI,etal.Reliability of diagnosing bacterial vaginosis is improved by standardized method of Gram stain interpretationsClinMicrobiol. 1991;29:297-301.

[49] Fredrick DN,FiedlerTI,ThomasKK, etal.Targetted PCR for detection of vaginal vaginal bacteria associated with bacterial vaginosis.JClin Microbiol.2007;45:3270-3276.

[50] SwelbergJ,Steiner JF, DeissF, et al.Comparison of single dose vs one week course of metronidazole for symptomatic of bacterial vaginosis.JMMA.1985;254:1046-1049.

[51] Harson JM,McGregor JA,HillierJI,etal.Metronizadole for bacterial vaginosis:A comparison of vaginal vs oral therapy.JReprod Med.2000;45:889-96. 
[52] SchmittC,SobelJD,MeriwetherBacterialvaginosis Treatment with clindamycin cream versus oral metronidazole.Obstet Gynaecol.1992;79:1023-26.

[53] SobelJ,PeipertJF,MacGregorJA,etal.Efficacy of clindamycin vaginal ovule(3- day treatment) vs clindamycin cream(7-day treatment)in bacterial vaginosis.Infect DisObstet Gynaecol.2000;9:9-15.

[54] SchwebiceJR,Desmond RA, A randomized trial of the duration of therapy with metronidazole plus or minus azithromycin for treatment of symptomatic bacterial vaginosis. Clin Infect Dis.2007;44:213-219.

[55] SwidsinskiA,Mendling W, Loening-BaukeV,et al. An adherent Gardnerellavaginalisbiofilimpersistant on the vaginal epithelium after standard therapy with oral metronidazole.Am J Obstet Gynaecol.2008;198:97.e6.

[56] SobelJD,Desquamative inflammatory vaginitis: A new subgroup of purulent vaginitis responsive to topical $2 \%$ clindamycin therapy. Am J ObstetGynaecol.1994;171: 1215-20.

[57] Nyrijesy P .Postmanupausalvaginitis.Curr Infect Dis Rep.2007;9:480-84.

[58] Palmer AR,LikisFE.Lactational atrophic vaginitis.J Midwifery Womens Health. 2003; 48:282-284. 